\title{
A rare Case of Cesarean Section Scar Ectopic Pregnancy Managed by Laparotomy
}

\author{
MEHER UN NISA ${ }^{1}$, NAZIA AYUB ${ }^{2}$, MEHREEN GUT ${ }^{3}$, NUDRAT ${ }^{4}$ \\ ${ }^{1}$ Professor of Obstetrics \& Gynaecology, PGMI/AMC/Lahore General Hospital, Lahore \\ ${ }^{2}$ Assistant of Obstetrics \& Gynaecology, PGMI/AMC/Lahore General Hospital, Lahore \\ ${ }^{3}$ Senior Registrar, Obstetrics \& Gynaecology, PGMI/AMC/Lahore General Hospital, Lahore \\ ${ }^{4} P G R$, Obstetrics \& Gynaecology, PGMI/AMC/Lahore General Hospital, Lahore \\ Correspondence to Prof. Meher un Nisa, E-mail:drmehersajawal@gmail.com Cell: 0322-4972794
}

\begin{abstract}
SUMMARY
Cesarean section scar ectopic pregnancy (CSEP) is defined as a pregnancy in which blastocyst is implanted within the scar of previous cesarean section. It is a rare form of ectopic but its frequency is increasing due to increasing rate of cesarean section. In this case, a 36 years old G3P2A0, married for 10 years, previous II C/Sections, LCB 7 years back, presented with ultrasound report of the viable pregnancy at $11+1$ weeks with gestational sac incorporating into previous scar of cesarean section. She was otherwise asymptomatic and stable. Serum Beta hCG was $73664.78 \mathrm{IU} / \mathrm{L}$. Laparotomy was done as an elective procedure. Dense adhesions were noted on opening the abdomen between anterior abdominal wall, uterus, bladder and gut. Scar ectopic pregnancy was excised after dissecting the adhesions. Her Serum Beta hCG 48 hour post-operatively was 1397.0 IU/L.Patient was discharged home on $2^{\text {nd }}$ post-operative day in good condition. She was counselled about risk of a recurrent scar ectopic and rupture of uterus in next pregnancy. Her serum Beta hCG declined to normal by 4 weeks post operatively.

Keywords: Cesarean section, scar ectopic, laparotomy
\end{abstract}

\section{INTRODUCTION}

Cesarean scar ectopic pregnancy (CSEP) is defined as implantation of blastocyst on the scar in the lower uterine segment. It is a rare condition but its rate is increasing due to rising rate of cesarean section. Its incidence is $1 / 1,800$ 2216 after cesarean section and $6.1 \%$ of all ectopic pregnancies ${ }^{1,2}$. First described by Larsen et al ${ }^{3}$ in 1978 and first diagnosis of CSEP by ultrasound was published in $1990^{4}$.

It is diagnosed by transvaginal and color flow Doppler ultrasound. The diagnostic criteria includes: gestational sac found in previous cesarean section scar, reduced thickness of myometrium between urinary bladder and site of previous scar, increased vascularization around the gestational sac and an empty uterine cavity and cervical canal. The pathophysiology of CSEP is not very clear. Risk factors include previous cesarean section wound dehiscence, poor closure of hysterotomy technique, in vitro fertilization (IVF), reduced time between previous cesarean and next pregnancy, etc 5 .

It is associated with maternal complications like scar rupture, hemorrhage and hysterectomies if not diagnosed and managed in time. Treatment options include conservative, medical, surgical and combination of medical and surgical ${ }^{6}$.

Surgical treatment may be done by hysteroscopy, laparoscopy or laparotomy depending upon size of gestational sac, presence of fetal cardiac activity and hemodynamic stability of the patient ${ }^{7}$. The uterine artery embolization may be done before surgery to reduce bleeding at surgery ${ }^{8}$. Recurrence rate recorded is $34 \%$ in one study and has no association with mode of treatment ${ }^{9}$.

Received on 23-02-2021

Accepted on 13-05-2021

\section{CASE REPORT}

Mrs. Mehwis Ramiz, 36 years of age, married for 10 years, G3P2A0, previous II C/Sections, last child born was 7 years back, having last menstrual period on 16 Oct 2020, presented on 2-01-2021 at 11+1 weeks in outdoor department of Department of Gynecology and Obstetrics, Lahore General Hospital. On ultrasound scan, the gestational sac was incorporating into previous scar of $\mathrm{C} /$ Section. The scar thickness at this early stage of pregnancy was $3.7 \mathrm{~mm} \&$ was bulging into the bladder. On this report, she was admitted for further management. She was otherwise asymptomatic and stable. Her vitals at the time of admission were: BP 110/70 mmHg, Pulse 104/min, temperature $98^{\circ}$ and respiratory rate 18 /minute. On examination Abdomen was soft and non-tender. On bimanual examination: vulva vagina were healthy, uterus was anteverted, 12 weeks size with restricted mobility and tenderness and fullness in anterior serum Beta hCG on 2/1/2021 (pre operatively) was 73664.78 IU/L, Covid-PCR was negative.

Ultrasound findings (9/12/2020): anteverted bulky uterus with gestational sac (CRL $13 \mathrm{~mm} / 7.3$ weeks) seen in lower uterine segment adjacent to the C-section scar with thinning of overlying myometrium. Single fetus with yolk sac seen with cardiac activity positive. Uterine cavity and cervical canal were empty. No adnexal mass or cyst seen. Final impression was cesarean section scar ectopic pregnancy.

Ultrasound (Figure 1) done on 31/12/2020 showed: Scar bulging into bladder, only $3.7 \mathrm{~mm}$ thick uterine wall in right lateral half of the lower uterine segment, fetal cardiac activity was positive, crown rumb length $(4.29 \mathrm{~cm})$ corresponding to 11 week plus 5 days. Uterine cavity was empty. 
Laparotomy was done on 4/1/2021 in Lahore General Hospital as an elective procedure. Bladder was catheterized. General anesthesia was given. Abdomen was opened by pfanensteil incision at the site of previous cesarean scar. Dense adhesions were noted on opening the abdomen between anterior abdominal wall, uterus, bladder and gut. Tubes and ovaries could not be seen due to dense adhesions which were dissected carefully, uterine fundas exposed, bladder separated by sharp dissection from body of uterus. While bladder was separated from uterus, previous uterine scar was identified, a bluish thin walled sac (Figure 2) appeared which was explored by dissecting deep around the sac. The sac along with placenta was removed with blood clots at the base.Haemostasis was secured. Drain placed at the site of ectopic. Abdomen closed in layers. Specimen of a well formed fetus with placenta (Figure 3) was sent for histopathology. Post-operatively patient remained stable.Foley'catheter and drain were removed on first postoperative day. Her Serum Beta hCG 48 hour postoperatively $(7 / 1 / 2021)$ was 1397.0 IU/L.Patient was discharged home on $2^{\text {nd }}$ post-operative day in good condition with follow up visit after one week for stitch removal and serum Beta hCG follow up. She was counselled about risk of a recurrent scar ectopic and rupture of uterus in next pregnancy. She was counselled for contraception use for at least 2-3 years and report to hospital early in case she conceives again. Serum Beta hCG returned to normal after 4 weeks postoperatively.

Figure 1: Scar ectopic (Ultrasound findings)

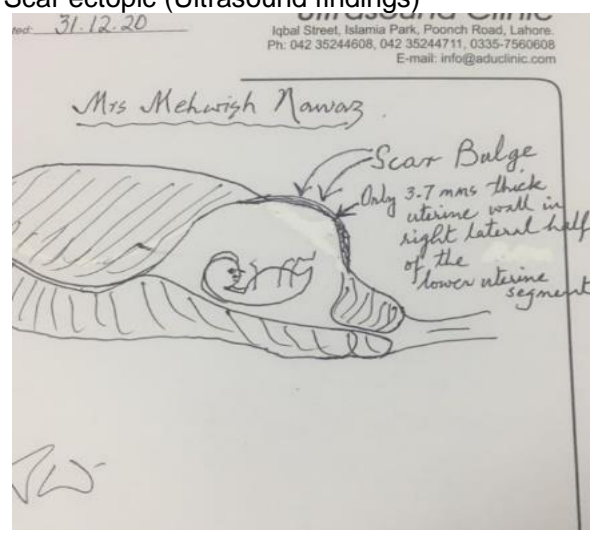

Figure 2: Scar ectopic as seen on laparotomy

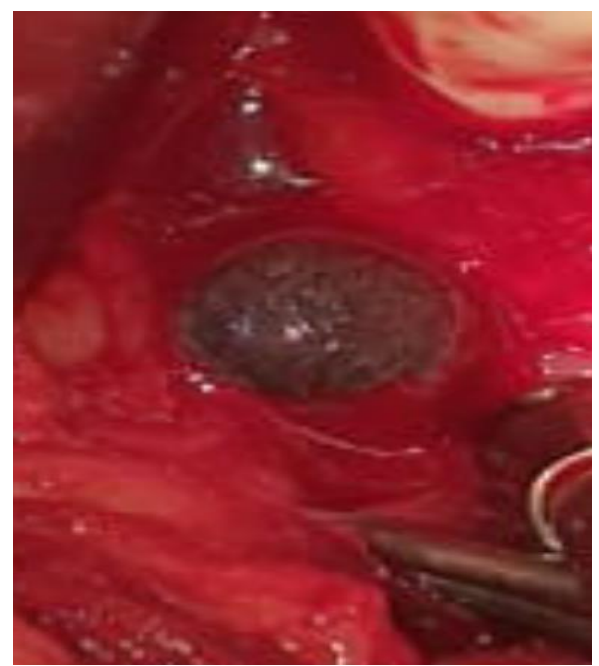

Figure 3: Scar ectopic after removing from $\mathrm{C} /$ Section scar

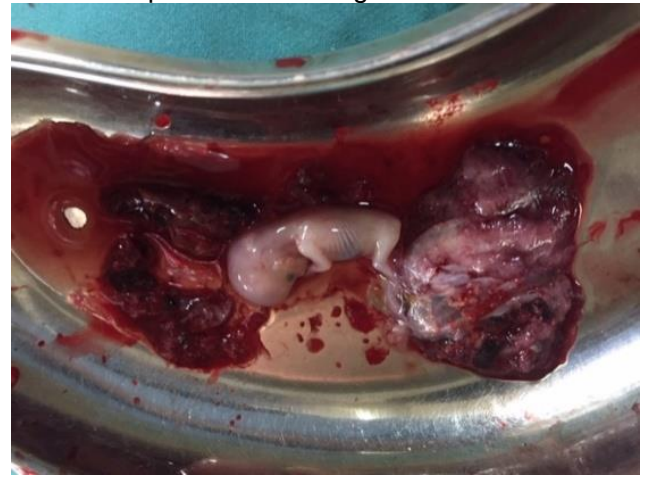

\section{DISCUSSION}

In this case report, the risk factors noted were previous 2 cesarean section along with history of cesarean sections done in a private set up with history of wound infection. There is controversy about the cause of scar ectopic pregnancy. It is reported to be due to implantation of blastocyst into the scar by invading myometrium via microscopic channels formed as a result of injury in previous cesarean section or other operations on uterus like myomectomy, hysterotomy and metroplasty ${ }^{10}$.

About $40 \%$ of patients with scar ectopic are asymtopatic ${ }^{11}$. Our patient was also asymptomatic. Scar ectopic can present with catastrophic complications like uterine rupture and life threatening hemorrhage, hysterectomy and loss of future fertility. ${ }^{11}$ The risk of rupture of scar pregnancy increases to 4 folds in case of single layer closure of uterus in previous cesarean section ${ }^{11,12}$.

Different management options of scar ectopic are mentioned in literature depending upon gestational age, level of serum Beta hCG and hemodynamic stability of patient. ${ }^{10,11,12}$ Elective surgery with removal of the sac and placenta and preservation of uterus is recommended if fertility is required. We followed this management protocol as patient wished to have more pregnancies, gestational age was more than 8 weeks (12 weeks on the day of surgery) and high level of serum Beta hCG (93664.IU/L) which must be less than $12000 \mathrm{IU} / \mathrm{L}$ to justify medical treatment ${ }^{13}$. 
In our case report, criteria for scar ectopic were fulfilled at ultrasound i.e. gestational sac located in lower uterine segment between bladder wall and lower segment anterior uterine wall which was thinned out with an empty uterine cavity. Difficulties were encountered during surgery due to adhesions which was expected due to history of wound infection in previous cesarean sections. The Beta hCG declined to normal by 4 weeks post operatively.

\section{CONCLUSION}

Early diagnosis and timely management in first trimester of CSEP can prevent complications like rupture, hemorrhage and hysterectomy.

Conflict of interest: Author claimed no conflict of interest

Author contributions; Nisa M: Drafted manuscript in terms of conceiving the idea, manuscript writing, editing and reviewing. Co-authors helped in data collection and editing the manuscript.

\section{REFERENCES}

1. KM Seow LW Huang YH Lin MY Lin YL Tsai JL Hwang.Cesarean scar pregnancy: issues in management Ultrasound Obstet Gynecol, 2004; 23:247 253.

2. Harjee R, Simula NK, Bedaiwy MA, Todd NJ. MANAGEMENT OF CESAREAN SCAR ECTOPIC PREGNANCIES. A COHORT STUDY. Fertility and Sterility. 2020 Sep 1; 114(3):e477.

3. Larsen JV, Solomon MH. Pregnancy in a uterine scar sacculus-an unusual cause of postabortal haemorrhage. A case report. S Afr Med J. 1978; 53:142-3.

4. Rempen A, Albert P. Diagnose und therapie einer in der sectionarbe implantierten Fruschwangerschaft. Geburtsh Perinat. 1990;194:46-8.
5. Gerday A, Lourtie A, Pirard C, Laurent P, Wyns C, Jadoul P, Squifflet JL, Dolmans MM, Van Gossum JP, Hammer F, Luyckx M. Experience With Medical Treatment of Cesarean Scar Ectopic Pregnancy (CSEP) With Local UltrasoundGuided Injection of Methotrexate. Frontiers in Medicine. 2020 ; 7 .

6. A Ash A Smith D Maxwell.Caesarean scar pregnancy. BJOG; 114: 253263

7. Najam S, Malik SE, Aqeel S, Rizwan N, Haider AR. Viable Caesarean Scar Pregnancy: A Case Report. Biomedica. 2020 Mar 1; 36(1).

8. Tumenjargal $\mathrm{A}$, Tokue $\mathrm{H}$, Kishi $\mathrm{H}$, Hirasawa $\mathrm{H}$, TaketomiTakahashi A, Tsushima Y. Uterine artery embolization combined with dilation and curettage for the treatment of cesarean scar pregnancy: efficacy and future fertility. Cardiovasc Intervent Radiol.2018; 41:1165-73.

9. Hoa NH, Thao NT, Tuyet HT. The Outcome of 40 Patients Becoming Pregnancy after Conservative Treatment of Cesarean Scar Pregnancy. Case Reports in Clinical Medicine. 2020 Dec 9; 9(12):376-84.

10. Junaid D, Chaudhry $S$, Usman M, Hussain R. CAESAREAN SCAR ECTOPIC PREGNANCY: A CASE SERIES. The Pakistan Journal of Medicine and Dentistry. 2018; 7(3):4-5.

11. Kashif A. Scar Ectopic Pregnancy. Journal of the College of Physicians and Surgeons Pakistan (JCPSP). 2020 Aug $1 ; 30(8): 884-5$.

12. Abbas AM, Ali SS, Michael A, Badran SA. Caesarean Scar Ectopic Pregnancy Complicated by Uterine Rupture at 10 Weeks Gestation. Journal of Gynecologic Surgery. 2017 Dec $1 ; 33(6): 261-3$.

13. 13. Bodur S, Özdamar Ö, Kılıç S, Gün I. The efficacy of the systemic methotrexate treatment in caesarean scar ectopic pregnancy: A quantitative review of English literature. Journal of Obstetrics and Gynaecology. 2015 Apr 3; 35(3):290-6. 